\title{
Individual attacks with generalized discrimination and inadequacy of some information measures
}

\author{
Alexey E. Rastegin \\ Department of Theoretical Physics, Irkutsk State University, Gagarin Bv. 20, Irkutsk 664003, Russia
}

\begin{abstract}
We accomplish studies of properly quantifying eavesdropper's information gain in individual attacks on the BB84 system of quantum key distribution. A noticeable sensitivity of conclusions to the choice of information measures is brightly revealed, when generalized state discrimination is used at the last stage. To realize her aims, Eve intrudes into the communication channel with some entangling probe. The Fuchs-Peres-Brandt (FPB) probe is known as a powerful individual attack on the BB84 protocol. In the simplest formulation, Eve uses the Helstrom scheme to distinguish output probe states. In conclusive eavesdropping, the unambiguous discrimination is utilized. In the intermediate scenario, she can apply a generalized discrimination scheme that interpolates between the Helstrom and unambiguous discrimination schemes. We analyze Eve's probe performance from the viewpoint of various measures for quantifying mutual information.
\end{abstract}

PACS numbers: 03.67.Dd, 03.65.Ta, 03.67.Hk

Keywords: BB84 protocol, Helstrom scheme, unambiguous discrimination, Rényi entropy, mutual information

\section{INTRODUCTION}

Today, quantum cryptography is considered to be a long-term solution to the problem of communication security [1-4]. It is one of emerging technologies based on new application of quantum phenomena [5 -7]. Due to famous Shor's results [8], we have obtained a lot of quantum algorithms for algebraic problems [9] including those with an impact on classical cryptology [10]. The BB84 protocol [11] is both the first and most known scheme for quantum key distribution. Another scheme of rather methodological interest is based on two non-orthogonal states [12]. One of basic problems in this field of investigations is to analyze vulnerabilities of quantum cryptography protocols. In principle, there are numerous scenarios of eavesdropper's activity. Individual attacks form the simplest and intuitively reasoned way to break a bit sequence shared due to quantum key distribution. Here, Eve intrudes into the channel and entangles each information carrier with her probe. After manipulations, altered carriers come to the receiver, whereas entangling probes are measured by Eve to recognize the original state of each carrier. The main question of information-theoretic origin is how to characterize quantitatively a performance of quantum cryptographic probes. Studies of individual attacks on systems of quantum cryptography were initiated in [13].

During quantum key distribution with eavesdropping, each of the three parties will obtain some string of bits. Treating such strings as random variables, a degree of dependence between two of them is usually measured by the mutual information. This approach used in [13] was motivated due to some results of the paper [14]. The authors of [15] have accomplished a detailed analysis of individual attacks. Together with the standard mutual information, the so-called Rényi mutual information was utilized in [15]. However, the treatment of mutual information quantifiers essentially depends on their properties. Such properties are closely related to the used definition of conditional entropies. There is no generally accepted definition of conditional entropy of the Rényi type [16, 17]. Existing approaches to this notion do not allow us to protect all the properties of the standard information functions. As is already mentioned in [18], some measures to quantify mutual information are inadequate in the context of quantum cryptography. This fact has been shown by comparing the two cases of state discrimination possible after the action of Eve's entangling probes. If Eve uses conclusive eavesdropping, then measures of mutual information of the Rényi type lead to wrong conclusions about a probe performance.

Conclusive eavesdropping is one of possible ways, in which Eve may discriminate states of her target qubit. There are intermediate discrimination schemes that interpolates between the Helstrom scheme and the unambiguous one. Such an analysis does not seem to have been previously recognized in the literature, but arises naturally in the problem of probe performance estimation. The aim of the present work is to study measures of mutual information from the viewpoint of such scenarios of eavesdropping. The paper is organized as follows. In Sect. II we briefly describe details of the Fuchs-Peres-Brandt probe for an individual attack on the BB84 protocol. Schemes of discrimination between two pure states are recalled as well. Section $\amalg$ iIs devoted to required information-theoretic notions with a short sketch of basic properties of the Rényi entropic functions. In Sect. IV. we apply both the Maassen-Uffink and majorization uncertainty relations to measurements of the considered form. Main findings are considered in Sect. V] In effect, one demonstrates a certain inadequacy of several $\alpha$-measures of information of the Rényi type to quantify Eve's probe performance. This feature is brightly revealed by consideration of the scenario with generalized state 
discrimination. This conclusion holds for the popular choice $\alpha=2$ as well as for larger orders that may be used in information functions. In Sect. VI] we conclude the paper.

\section{DETAILS OF THE FPB PROBE}

In this section, we first recall the Fuchs-Peres-Brandt probe for an individual attack on the BB84 protocol. The second part of this section briefly describes existing schemes of discrimination between two pure states of a qubit. The problem of optimization of probe characteristics was originally examined by Fuchs and Peres [19]. They showed numerically that the optimal detection method for a two-level system can be obtained with a two-dimensional probe. Brandt [20, 21] later noted that the obtained probe for attacking the BB84 scheme can be realized with a single CNOT gate. Following [22, 23], this probe will be referred to as the Fuchs-Peres-Brandt (FPB) probe. The wellknown analysis of [15] is related to the case, in which the error-discard method is used as a reconciliation procedure. When Alice and Bob use other reconciliation methods, the FPB probe is generally not optimal [24]. We will assume that Bob's detectors and Eve's detectors have not failed at all. In this sense, a discussed situation is idealized. It is natural that security analysis depends on the assumptions used to schematize a practical setup. Concerning the BB84 protocol, important steps in security proofs were accomplished in the papers [25 27]. Individual attacks have been analyzed in various respects [28 30]. Security of quantum key distribution against collective attacks was examined in [31].

In the BB84 scheme, Alice and Bob use the two polarization bases, $\{|h\rangle,|v\rangle\}$ and $\{|r\rangle,|\ell\rangle\}$. With respect to the horizontal polarization, the kets $|r\rangle$ and $|\ell\rangle$ of the diagonal basis relate to the angles $\pi / 4$ and $3 \pi / 4$, respectively. In each bit interval Alice sends a single photon prepared accordingly. Intercepting this photon, Eve inputs it as the control qubit into her CNOT gate acting in the basis of kets

$$
\begin{aligned}
& |0\rangle=\cos (\pi / 8)|h\rangle+\sin (\pi / 8)|v\rangle, \\
& |1\rangle=-\sin (\pi / 8)|h\rangle+\cos (\pi / 8)|v\rangle .
\end{aligned}
$$

Eve prepares her own probe photon in the initial state

$$
\left|t_{i n}\right\rangle=c|+\rangle+s|-\rangle
$$

where $c=\sqrt{1-2 P_{E}}, s=\sqrt{2 P_{E}}$, and

$$
| \pm\rangle=\frac{|0\rangle \pm|1\rangle}{\sqrt{2}} .
$$

The sense of the parameter $P_{E} \in[0 ; 0.5]$ is clarified as follows. Once Alice and Bob have produced some string, they select a sample of sufficient size and publicly announce the values of those bits. To detect Eve's activity, Alice and Bob calculate the fraction of bits which disagree. This fundamental parameter is called the bit error rate.

The state (3) is used as the target qubit of Eve's CNOT gate controlled by sent Alice's qubit. Here, we will express the gate output in the form, in which the first qubit is represented in a proper basis. Let us introduce subnormalized vectors

$$
\begin{aligned}
& \left|t_{ \pm}\right\rangle=c|+\rangle \pm \frac{s}{\sqrt{2}}|-\rangle, \\
& \left|t_{E}\right\rangle=\frac{s}{\sqrt{2}}|-\rangle .
\end{aligned}
$$

When Alice uses the basis $\{|h\rangle,|v\rangle\}$, Eve's CNOT gate acts as

$$
\begin{aligned}
|h\rangle \otimes\left|t_{i n}\right\rangle \longmapsto|h\rangle \otimes\left|t_{+}\right\rangle+|v\rangle \otimes\left|t_{E}\right\rangle, \\
|v\rangle \otimes\left|t_{i n}\right\rangle \longmapsto|v\rangle \otimes\left|t_{-}\right\rangle+|h\rangle \otimes\left|t_{E}\right\rangle .
\end{aligned}
$$

For states of the basis $\{|r\rangle,|\ell\rangle\}$, we similarly get

$$
\begin{aligned}
& |r\rangle \otimes\left|t_{i n}\right\rangle \longmapsto|r\rangle \otimes\left|t_{+}\right\rangle-|\ell\rangle \otimes\left|t_{E}\right\rangle, \\
& |\ell\rangle \otimes\left|t_{i n}\right\rangle \longmapsto|\ell\rangle \otimes\left|t_{-}\right\rangle-|r\rangle \otimes\left|t_{E}\right\rangle .
\end{aligned}
$$

The vectors $\left|t_{ \pm}\right\rangle$and $\left|t_{E}\right\rangle$ in the above formulas are subnormalized. So, their squared norms are related to the corresponding probabilities. Calculating the inner product $\left\langle t_{+} \mid t_{-}\right\rangle=1-3 P_{E}$, we further restrict a consideration to the range $P_{E} \in[0 ; 1 / 3]$. 
Suppose that Bob measures the received photon just in the basis that Alice has employed. With no eavesdropping, his outcomes will match what Alice sent. After a sufficiently long session of sending photons, Bob has chosen the right basis in a half of cases. Alice and Bob then discard all cases, in which Bob has taken the wrong basis. Hence, they share the sifted bits that should be error-free without eavesdropping. It is seen from the formulas (7)-(10) that Bob's outcome will sometimes be opposite to what Alice has sent. Such cases are described by the terms which involve the subnormalized vector $\left|t_{E}\right\rangle$. As its squared norm is equal to $P_{E}$, the latter term gives a fraction of mismatches in those bits that have been used to test eavesdropping. To learn shared bit values of Alice and Bob, Eve should distinguish between the subnormalized outputs $\left|t_{+}\right\rangle$and $\left|t_{-}\right\rangle$of the target qubit. At this stage, she could apply suitable schemes of quantum states discrimination.

In general, different quantum states cannot be perfectly discriminated. In the approach developed by Helstrom [32, 33], the optimal measurement is built to minimize the average probability of erroneous answer. Another method is known as unambiguous state discrimination as well as the IDP scheme due to Ivanovich [34], Dieks [35], and Peres [36]. From a general perspective, unambiguous discrimination was discussed in [37]. Its principal point is possibility of inconclusive answer, which allows us to avoid the error of misidentification. The version of the B92 scheme with unambiguous discrimination on Bob's side was examined in [13]. Of course, more general strategies of discrimination could be assumed. Concerning the case of linearly independent pure states, such strategies were addressed in [38, 39]. The authors of [40] extended this approach to mixed quantum states. In the following, we briefly recall the generalized scheme of discrimination of two non-orthogonal pure states.

Concerning the BB84 protocol, the following fact should be mentioned. There exist a version of the protocol, in which Alice and Bob extract a secret key from mismatched measurements [41]. In these cases, Alice's and Bob's actual bases do not coincide. In the standard version, results of such measurements are completely discarded. As is shown in [41], the legitimate users of the channel can process results of mismatched measurements to share secret key. The procedures of information reconciliation and privacy amplification are realized similarly to Shor and Preskill [25]. The authors of [41] showed that the key rates available from matched and mismatched measurements cannot be simultaneously positive. An essential ingredient of this proof is based on the Maassen-Uffink uncertainty relation [42]. Another important observation is that the participants cannot extract a secret key, when outcomes of mismatched measurements have equal probabilities. The FPB probe is such that generated error probabilities are the same with respect to the two bases. For this reason, we further focus on the standard treatment of the BB84 protocol.

The most direct way to discriminate pure states uses two projectors being a particular case of the Helstrom scheme. More sophisticated approach is known as unambiguous state discrimination. In the context of individual attacks, this approach was examined in [43]. As is pointed out in [22], the existence of inconclusive answer leads to more complicated character of performance of the FPB probe. Unambiguous state discrimination plays an important role in source-side attacks on decoy-state quantum key distribution with the source realized through a weak coherent state [44]. It is also possible to use a generalized discrimination scheme that interpolates between the Helstrom and unambiguous discrimination schemes 38 -40].

To learn an Alice's qubit, Eve should distinguish between $\left|t_{+}\right\rangle$and $\left|t_{-}\right\rangle$. Recall that these states are symmetric with respect the basis $\{|+\rangle,|-\rangle\}$. Further, we will refer just to this basis. Using the parameter $\theta$, we consider the states $\left|\theta_{+}\right\rangle$and $\left|\theta_{-}\right\rangle$expressed as

$$
\left|\theta_{+}\right\rangle=\left(\begin{array}{c}
\cos \theta \\
\sin \theta
\end{array}\right), \quad\left|\theta_{-}\right\rangle=\left(\begin{array}{c}
\cos \theta \\
-\sin \theta
\end{array}\right)
$$

Their inner product is equal to $\left\langle\theta_{+} \mid \theta_{-}\right\rangle=\cos 2 \theta$. Assuming $\theta \in(0 ; \pi / 4)$, we focus on non-identical and non-orthogonal states. Due to the results of Davies [45], one can restrict a consideration to rank-one measurement operators. We will express them in terms of three unit kets, namely

$$
\left|\gamma_{+}\right\rangle=\left(\begin{array}{c}
\sin \gamma \\
\cos \gamma
\end{array}\right), \quad\left|\gamma_{-}\right\rangle=\left(\begin{array}{c}
\sin \gamma \\
-\cos \gamma
\end{array}\right), \quad\left|\gamma_{?}\right\rangle=|+\rangle=\left(\begin{array}{l}
1 \\
0
\end{array}\right)
$$

where $\gamma$ is some angle. The latter is chosen so that $\left|\gamma_{+}\right\rangle$is closer to $\left|\theta_{+}\right\rangle$and $\left|\gamma_{-}\right\rangle$is closer to $\left|\theta_{-}\right\rangle$. The measurements operators are proportional to the operators $\left|\gamma_{ \pm}\right\rangle\left\langle\gamma_{ \pm}\right|$and $\left|\gamma_{?}\right\rangle\left\langle\gamma_{?}\right|$. To provide the completeness relation,

$$
\mathrm{M}_{+}+\mathrm{M}_{-}+\mathrm{M}_{?}=\mathbb{1}
$$

coefficients should obey certain conditions. Here, we present only the final expressions,

$$
\mathbf{M}_{ \pm}=\frac{1}{1+\cos 2 \gamma}\left|\gamma_{ \pm}\right\rangle\left\langle\gamma_{ \pm}\left|, \quad \mathbf{M}_{?}=\frac{2 \cos 2 \gamma}{1+\cos 2 \gamma}\right| \gamma_{?}\right\rangle\left\langle\gamma_{?}\right|
$$


For each of the two inputs $\left|\theta_{+}\right\rangle$and $\left|\theta_{-}\right\rangle$, one may give three outcomes, namely the success answer, the erroneous one and the inconclusive one. If the inputs have equal prior probabilities, then the average probabilities are written as

$$
Q_{S}=\frac{\sin ^{2}(2 \theta+\phi)}{1+\cos 2 \gamma}, \quad Q_{E}=\frac{\sin ^{2} \phi}{1+\cos 2 \gamma}, \quad Q_{?}=\frac{2 \cos 2 \gamma \cos ^{2} \theta}{1+\cos 2 \gamma} .
$$

Here, we introduce auxiliary angle $\phi$ such that $0 \leq \phi \leq \pi / 4-\theta$ and $\gamma=\theta+\phi$. It should be pointed out that the above expressions saturate the lower bound [38]

$$
Q_{E} \geq \frac{1}{2}\left(1-Q_{?}-\sin 2 \theta \sqrt{1-\frac{Q_{?}}{\cos ^{2} \theta}}\right)
$$

The measurement operators (14) are optimal with respect to the symmetric case of equiprobable inputs. For more general cases, see the papers [38 40]. We ask for a performance of the FPB probe, when Eve uses the described intermediate scheme of state discrimination.

In the following, we will refer to the particular cases of the above formulas. In the Helstrom scheme, we take $\phi=\pi / 4-\theta$, whence $Q_{?}=0$ and

$$
\begin{aligned}
& Q_{S}=\frac{1+\sin 2 \theta}{2}=\frac{1+\sqrt{1-\left\langle\theta_{+} \mid \theta_{-}\right\rangle^{2}}}{2}, \\
& Q_{E}=\frac{1-\sin 2 \theta}{2}=\frac{1-\sqrt{1-\left\langle\theta_{+} \mid \theta_{-}\right\rangle^{2}}}{2} .
\end{aligned}
$$

The IDP scheme is obtained for $\phi=0$ and $\gamma=\theta$. In this case, we have $Q_{?}=\cos 2 \theta=\left\langle\theta_{+} \mid \theta_{-}\right\rangle, Q_{S}=1-Q_{\text {? }}$ and $Q_{E}=0$. If we restrict a consideration to the error-free sifted bits, then the quantity $Q_{\text {? }}$ gives a fraction of inconclusive outcomes in Eve's results. Conclusive eavesdropping was originally considered by Brandt 43. Thus, POVM measurements of the form (14) can be used for generalized discrimination interpolating between the Helstrom and IDP schemes.

\section{INFORMATION-THEORETIC FUNCTIONS OF THE RÉNYI TYPE}

In this section, we recall some information-theoretic notions based on the Rényi entropies. Quantum key distribution allows Alice and Bob to obtain two identical copies of a random and secret sequence of bits. Intruding into a communication channel, Eve tries to recognize the secret sequence. During the process, each of the three parties will obtain some string of bits. The three strings are typically treated as binary random variables [13]. To measure a dependence between two random variables, one utilizes the concept of mutual information.

Let discrete variable $X$ take values on some finite set, and let $\{p(x)\}$ be the corresponding probability distribution. The Shannon entropy of $X$ is defined as [46]

$$
H(X):=-\sum_{x} p(x) \log p(x)
$$

The range of summation is usually clear from the context. The logarithm in (19) is taken to the base 2 . If $Y$ is another random variable, then the joint entropy $H(X, Y)$ is defined by substituting joint probabilities $p(x, y)$ into (19).

The concept of mutual information is connected with conditional entropies. The conditional form is commonly used in information theory [46] as well as in applied disciplines. The standard conditional entropy is defined by

$$
H(X \mid Y):=\sum_{y} p(y) H(X \mid y)=-\sum_{x} \sum_{y} p(x, y) \log p(x \mid y)
$$

Together with Bayes' rule $p(x \mid y)=p(x, y) / p(y)$, we used the particular function

$$
H(X \mid y)=-\sum_{x} p(x \mid y) \log p(x \mid y) .
$$

It follows from concavity that $H(X \mid Y, Z) \leq H(X \mid Y)$. In other words, conditioning on more can only reduce the conditional entropy. The quantity (20) leads to one of widely used information distances [47]. The chain rule is very important [46]

$$
H(X, Y)=H(X \mid Y)+H(Y)=H(Y \mid X)+H(X)
$$


The expression $H(X \mid Y)=H(X, Y)-H(Y)$ reflects remaining lack of knowledge about $X$ at the given $Y$.

The mutual information aims to measure how much information $X$ and $Y$ have in common [46], namely

$$
I(X, Y):=H(X)+H(Y)-H(X, Y) .
$$

The quantity (23) is clearly symmetric in entries. It follows from the chain rule that

$$
I(X, Y)=H(X)-H(X \mid Y)=H(Y)-H(Y \mid X) .
$$

So, the mutual information shows a reduction in the uncertainty of one random variable due to knowledge of others [46]. In the context of quantum cryptography, the notion (23) was first applied in [13]. Known extensions of the above concepts to entropic functions of the Rényi type lose some of the standard information-theoretic properties. For a general discussion of distinguishability measures in quantum information science, see [48, 49] and references therein. Entropic functions in application to quantum theory are reviewed in [50].

Overall, the standard information functions can be treated in operational terms. Moreover, the same formal expressions lead to regular information functions of density matrices. It is important since the case of classicalquantum-quantum-correlations underlies an analysis of secret-key distillation [51]. In particular, one uses (24) to characterize the amount of information accessible when a sender encodes symbols via quantum states. The accessible information is bounded from above due to the Holevo theorem (see, e.g., subsection 12.1.1 of [5]). In the context of quantum cryptography, the Holevo quantity plays an important role in the Devetak-Winter formula [51]. The so-called Devetak-Winter rate shows the asymptotic performance of a protocol with one-way classical communication under collective attacks [4]. At the same time, the use of the mutual information (24) per se allows one to examine security against collective attacks [31]. The optimal collective attack in the "device-independent" security scenario was built in [52]. When Eve utilizes individual attacks, the security bound for one-way postprocessing can be given according to Csiszár and Körner [14]. It is sufficient for our aims to restrict a consideration to individual attacks. In more detail, different types of attacks and related security characteristics are reviewed in section III of [4].

Rényi entropies form an important family of one-parametric extensions of the Shannon entropy (19). For $0<\alpha \neq 1$, the Rényi $\alpha$-entropy is defined as [53]

$$
R_{\alpha}(X):=\frac{1}{1-\alpha} \log \left(\sum_{x} p(x)^{\alpha}\right) .
$$

It does not increase with growth of $\alpha[53$. If $p(x)=1 / N$ for all $x$, then the entropy (25) reaches its maximal value equal to $\log N$. In the limit $\alpha \rightarrow 1$, the right-hand side of (25) reduces to (19). The joint $\alpha$-entropy $R_{\alpha}(X, Y)$ is defined according to (25) by substituting the joint probabilities. Special choices of the order $\alpha$ are widely used in the literature. In the limit $\alpha \rightarrow \infty$, we obtain the min-entropy

$$
R_{\infty}(X)=-\log (\max p(x)) .
$$

The case $\alpha=2$ gives the so-called collision entropy, viz.

$$
R_{2}(X)=-\log \left(\sum_{x} p(x)^{2}\right) .
$$

For $\alpha \in[0 ; 1]$, the Rényi entropy is concave independently of the effective dimensionality [54]. Convexity properties of $R_{\alpha}(X)$ with orders $\alpha>1$ actually depend on dimensionality of probabilistic vectors [50, 55]. In particular, the binary Rényi entropy is concave for $\alpha \in[0 ; 2][55]$.

To quantify mutual information via functions of the Rényi type, the corresponding conditional entropies should be used. There is no generally accepted definition of conditional Rényi's entropy [16]. This variety reflect the fact that generalized entropic functions lack some of important properties of regular ones. There are several ways to treat the standard information functions in operational terms. It is hardly able to keep all of them, when generalized functions are dealt with. First, we recall the quantity [56, 57]

$$
R_{\alpha}^{(1)}(X \mid Y):=\sum_{y} p(y) R_{\alpha}(X \mid y),
$$

where

$$
R_{\alpha}(X \mid y):=\frac{1}{1-\alpha} \log \left(\sum_{x} p(x \mid y)^{\alpha}\right) .
$$

Despite of certain similarity between (20) and (28), the treatment of $R_{\alpha}^{(1)}(X \mid Y)$ as conditional entropy is not completely legitimate. Basic properties of (28) are considered in [56, 57]. More special properties of the above conditional entropy 
with some applications were addressed in 58, 59]. The entropy (28) has been used in formulating the uncertainty principle in successive measurements [60, 61]. This scenario is very close to that is dealt with in quantum key distribution with eavesdropping.

It turned out that the conditional entropy (28) does not share the chain rule. Instead of (28), other definitions of conditional Rényi's entropy were proposed [16]. The authors of [62] proposed the conditional entropy written as

$$
R_{\alpha}^{(2)}(X \mid Y):=R_{\alpha}(X, Y)-R_{\alpha}(Y) .
$$

Here, the chain rule is posed by definition. However, the right-hand side of (30) cannot be re-expressed similarly to (28). The authors of [17] reconsidered the notion of conditional Rényi's entropy including five known suggestions and also Arimoto's version. The first and second of them are, respectively, defined as (28) and (30). The forth definition from the list of [17] is of interest due to its fine properties. For $0<\alpha \neq 1$, it is expressed as

$$
R_{\alpha}^{(4)}(X \mid Y):=\frac{1}{1-\alpha} \log \left(\sum_{y} p(y) \sum_{x} p(x \mid y)^{\alpha}\right) .
$$

The third and fifth definitions from the list of [17] are inconsistent with the standard conditional entropy (20). Further, we do not consider these definitions. It seems that Arimoto's conditional Rényi entropy has found less attention than it deserves [17]. One of its promising properties is the direct link to the corresponding quantum conditional entropy. The latter notion naturally follows from the so-called "sandwiched" $\alpha$-divergence of the Rényi type. Such quantum divergences were proposed and motivated in 63, 64]. On the other hand, Arimoto's conditional Rényi entropy has more complicated functional form than the entropies (28), (301) and (31).

The conditional entropy (28) leads to the mutual information quantifier that has found use in studying individual attacks on quantum cryptographic systems [15, 21 23, 43]. It is often treated without a complete theoretical background. The standard mutual information has some useful representations, each of which may give us an inspiration to define an $\alpha$-mutual information measure. In principle, the literature offers several approaches to accomplish such generalization [65]. An unsophisticated approach to fit the concept of $\alpha$-information merely takes the source from (24). By an analogy with the formula (24), we can define the $\alpha$-mutual information

$$
I_{\alpha}^{(1)}(X, Y):=R_{\alpha}(X)-R_{\alpha}^{(1)}(X \mid Y) .
$$

It is often interpreted as the Rényi $\alpha$-measure of mutual information. With the choice $\alpha=2$, this quantity has been adopted as a measure of probe performance in individual attacks on quantum cryptographic schemes [15, 2123, 43]. Thus, the quantity (32) has found use in applied disciplines, despite of incomplete status from the theoretical viewpoint. When $\alpha \neq 1$, the quantity (32) differs from (24) in some important respects. First, the function (32) is not generally symmetric in its entries. Second, the parameter $\alpha$ runs a continuum of values, whence we should make a proper choice of its value. Advantages of the approach with generalized entropic functions include a possibility to vary the used parameter [57]. On the other hand, the conditional $\alpha$-entropy (28) does not share the chain rule. So, the right-hand side of (32) is something similar to (24) but is not similar to (23). One fails with interpreting (32) as a reduction in the uncertainty of one random variable due to knowledge of others. For completeness of the presentation, we will also consider the quantities

$$
\begin{aligned}
& I_{\alpha}^{(2)}(X, Y):=R_{\alpha}(X)-R_{\alpha}^{(2)}(X \mid Y)=R_{\alpha}(X)+R_{\alpha}(Y)-R_{\alpha}(X, Y), \\
& I_{\alpha}^{(4)}(X, Y):=R_{\alpha}(X)-R_{\alpha}^{(4)}(X \mid Y) .
\end{aligned}
$$

Here, we actually rewrite the right-hand side of (24) with the corresponding Rényi entropies. The quantity (33) is obviously symmetric in entries. In both the cases, the limit $\alpha \rightarrow 1$ leads to the standard mutual information (24).

Thus, information functions of the Rényi type are obtained in several ways. This non-uniqueness is revealed in many questions including characteristics of quantum coherence [66, 67]. One must be very careful in interpretation of (32)-(34), especially if only one value $\alpha \neq 1$ is involved. In the following, we will use the term " $\alpha$-measures of mutual information". However, these quantities are not fully legitimate measures of mutual information. This fact was shown from the viewpoint of analyzing a performance of probes in individual attacks on the BB84 protocol [18]. It was assumed in [18] that Eve can utilize only the two schemes of state discrimination. The first of them is due to Helstrom 32, 33], and the second one is commonly referred to as unambiguous discrimination independently developed by in [34 36]. Focusing on the conclusive eavesdropping allows us to reveal a noticeable inadequacy of (32) served as the measure of mutual information. This conclusion remains valid with more general scenarios of quantum state discrimination on Eve's side. 


\section{ENTROPIC UNCERTAINTY RELATIONS FOR GENERALIZED DISCRIMINATION}

We will also use some entropic uncertainty bounds for measurements of the form (14). In the case of finitedimensional systems, entropic uncertainty relations were developed due to Deutsch [68] and Maassen and Uffink [42]. Many important results obtained in this way are reviewed in [69, 70]. Let us consider orthonormal bases $\mathcal{G}=\left\{\left|g_{i}\right\rangle\right\}$ and $\mathcal{G}^{\prime}=\left\{\left|g_{j}^{\prime}\right\rangle\right\}$ in $d$ dimensions. If the pre-measurement state is described by $d \times d$ matrix $\rho$, then the generated probabilities are equal to $\left\langle g_{i}|\boldsymbol{\rho}| g_{i}\right\rangle$ and $\left\langle g_{j}^{\prime}|\boldsymbol{\rho}| g_{j}^{\prime}\right\rangle$, respectively. Substituting these probabilities into (25), we have the corresponding entropies $R_{\alpha}(\mathcal{G} ; \boldsymbol{\rho})$ and $R_{\alpha}\left(\mathcal{G}^{\prime} ; \boldsymbol{\rho}\right)$. The Maassen-Uffink uncertainty relation is posed as follows. One should take the unitary matrix $\mathrm{W}$ with entries $w_{i j}=\left\langle g_{i} \mid g_{j}^{\prime}\right\rangle$. For $1 / \alpha+1 / \beta=2$, we have

$$
R_{\alpha}(\mathcal{G} ; \boldsymbol{\rho})+R_{\beta}\left(\mathcal{G}^{\prime} ; \boldsymbol{\rho}\right) \geq-2 \log s_{\max }
$$

where

$$
s_{\max }:=\max _{i j}\left|w_{i j}\right| .
$$

Maassen and Uffink presented the relation with Shannon entropies, viz. [42]

$$
H(\mathcal{G} ; \boldsymbol{\rho})+H\left(\mathcal{G}^{\prime} ; \boldsymbol{\rho}\right) \geq-2 \log s_{\max },
$$

but their method is actually sufficient for (35). It must be stressed that the result (37) was initially conjectured by Kraus [71]. The author of [72] mentioned how the above relations can be applied to a single POVM. In effect, the given POVM measurement can be realized as a projective one with different Naimark extensions. Adding third component to kets of the set $\left\{\left|\gamma_{ \pm}\right\rangle,\left|\gamma_{?}\right\rangle\right\}$, we can build an orthonormal basis with the unitary Gramian matrix of size 3. The resulting vectors are written as

$$
\left|\widetilde{\gamma}_{ \pm}(\varphi)\right\rangle=\frac{1}{\sqrt{1+\eta}}\left(\begin{array}{c}
\sin \gamma \\
\pm \cos \gamma \\
\sqrt{\eta} e^{i \varphi}
\end{array}\right), \quad\left|\widetilde{\gamma}_{?}(\varphi)\right\rangle=\frac{1}{\sqrt{1+\eta}}\left(\begin{array}{c}
\sqrt{2 \eta} \\
0 \\
-\sqrt{1-\eta} e^{i \varphi}
\end{array}\right)
$$

where $\eta=\cos 2 \gamma$. The phase factor $e^{i \varphi}$ shows a unitary freedom in the ancillary one-dimensional space. Varying $\varphi$, we obtain different extensions of the original POVM. We now apply (35) and (37) to the considered POVM by inspecting matrix elements of the form $\left\langle\widetilde{\gamma}_{i}(\varphi) \mid \widetilde{\gamma}_{j}\left(\varphi^{\prime}\right)\right\rangle$, so that

$$
s_{\max }\left(\varphi, \varphi^{\prime}\right):=\max _{i j}\left|\left\langle\widetilde{\gamma}_{i}(\varphi) \mid \widetilde{\gamma}_{j}\left(\varphi^{\prime}\right)\right\rangle\right|
$$

As is noted in [72], we can further optimize the lower bound $-2 \log s_{\max }\left(\varphi, \varphi^{\prime}\right)$ with respect to freely variable parameters. Calculations are somewhat long, though simple in matter. We refrain from presenting the details here. The uncertainty relation for the POVM $\mathcal{M}=\left\{\mathrm{M}_{+}, \mathrm{M}_{-}, \mathrm{M}_{?}\right\}$ reads as

$$
R_{\alpha}(\mathcal{M} ; \boldsymbol{\rho})+R_{\beta}(\mathcal{M} ; \boldsymbol{\rho}) \geq 2 \log f(\eta)
$$

where we put the function

$$
f(\eta):= \begin{cases}\frac{1+\eta}{1-\eta}, & \text { if } \eta \in[0 ; 0.2] \\ \sqrt{\frac{2-\eta}{1-\eta}}, & \text { if } \eta \in[0.2 ; 0.5] \\ \sqrt{\frac{2-\eta}{\eta}}, & \text { if } \eta \in[0.5 ; 1] .\end{cases}
$$

The authors of [73] proposed an improvement of (37). In our notation, their result is expressed as

$$
H(\mathcal{G} ; \boldsymbol{\rho})+H\left(\mathcal{G}^{\prime} ; \boldsymbol{\rho}\right) \geq-2 \log s_{\max }+\left(1-s_{\max }\right) \log \left(\frac{s_{\max }}{s_{2}}\right)
$$

where $s_{2}$ is the second largest value among moduli $\left|w_{i j}\right|$ of entries $w_{i j}=\left\langle g_{i} \mid g_{j}^{\prime}\right\rangle$. Applying (42) to the considered POVM, we have

$$
H(\mathcal{M} ; \boldsymbol{\rho}) \geq \log f(\eta)+\frac{\eta U(0.2-\eta)}{2(1+\eta)} \log \left(\frac{1-\eta}{4 \eta}\right)
$$


By $U(x)$, we denote here the unit step function, which is 0 for $x<0$ and 1 for $x>0$. In the right-hand side of (43), the correction term is nonzero only for $\eta<0.2$. It turns out that, for $\eta>0.2$, optimizing the term $-2 \log s_{\max }\left(\varphi, \varphi^{\prime}\right)$ results in $s_{\max }=s_{2}$.

Although the Maassen-Uffink uncertainty relation usually provides a very good estimation, majorization uncertainty relations are sometimes stronger 74 76]. Here, some facts of matrix analysis should be recalled. For two integers $m, n \geq 1$, the symbol $\mathbb{M}_{m \times n}(\mathbb{C})$ denotes the space of all $m \times n$ complex matrices. For any $A \in \mathbb{M}_{m \times n}(\mathbb{C})$, the square matrices $\mathrm{A}^{\dagger} \mathrm{A}$ and $\mathrm{AA}^{\dagger}$ have the same nonzero eigenvalues. Taking the square root of these eigenvalues, one gets nonzero singular values $\sigma_{j}(\mathrm{~A})$. The largest of singular values gives the spectral norm $\|\mathrm{A}\|_{\infty}$ of $\mathrm{A}$. To the given unitary matrix $\mathrm{W}$, we assign the set of all its submatrices of class $k$ defined by

$$
\mathcal{S U B}(\mathrm{W}, k):=\left\{\mathrm{A} \in \mathbb{M}_{r \times r^{\prime}}(\mathbb{C}): r+r^{\prime}=k+1, \mathrm{~A} \text { is a submatrix of } \mathrm{W}\right\} .
$$

The majorization relations of [74, 76] are formulated in terms of positive quantities

$$
\zeta_{k}:=\max \left\{\|\mathrm{A}\|_{\infty}: \mathrm{A} \in \mathcal{S U B}(\mathrm{W}, k)\right\}
$$

Majorization relations of the tensor-product type [74, 75] are written with a probability vector $\omega^{\prime}$ such that

$$
p \otimes q \prec \omega^{\prime},
$$

where $p_{i}=\left\langle g_{i}|\boldsymbol{\rho}| g_{i}\right\rangle$ and $q_{j}=\left\langle g_{j}^{\prime}|\boldsymbol{\rho}| g_{j}^{\prime}\right\rangle$. The authors of [74] showed that the relation (46) holds for

$$
\omega^{\prime}=\left(\xi_{1}, \xi_{2}-\xi_{1}, \ldots, \xi_{d}-\xi_{d-1}\right), \quad \xi_{k}=\frac{\left(1+\zeta_{k}\right)^{2}}{4} .
$$

It follows from (47) that, for all $\alpha>0$, the sum of two $\alpha$-entropies obeys

$$
R_{\alpha}(p)+R_{\alpha}(q) \geq R_{\alpha}\left(\omega^{\prime}\right) .
$$

Majorization relations of the direct-sum type presented in [76] are based on the relation

$$
p \oplus q \prec\{1\} \oplus \omega .
$$

Generated probabilistic vectors obey (49) with $\omega=\left(\zeta_{1}, \zeta_{2}-\zeta_{1}, \ldots, \zeta_{d}-\zeta_{d-1}\right)$ [76]. The majorization relation (49) further implies that

$$
R_{\alpha}(p)+R_{\alpha}(q) \geq \begin{cases}R_{\alpha}(\omega), & \text { for } 0<\alpha \leq 1 \\ \frac{2}{1-\alpha} \log \left(\frac{1}{2}+\frac{1}{2} \sum_{i} \omega_{i}^{\alpha}\right), & \text { for } 1<\alpha<\infty\end{cases}
$$

Overall, the elements $\zeta_{k}$ are expressed due to (44) and (45) with the unitary matrix W $=\left[\left[\left\langle g_{i} \mid g_{j}^{\prime}\right\rangle\right]\right]$. Although the subscript $k$ in (45) runs from 1 up to $d^{2}-1$, the condition of unitarity leads to $\zeta_{d}=1$ and, herewith, $\zeta_{k}=1$ for $k \geq d$. For $0<\alpha \leq 1$, one certainly has $R_{\alpha}(\omega) \geq R_{\alpha}\left(\omega^{\prime}\right)$ [76]. For $\alpha>1$, we should keep in mind both the formulas (48) and (50). We apply these relations to the POVM $\mathcal{M}=\left\{M_{+}, M_{-}, M_{?}\right\}$ with different Naimark extensions. By doing some calculations, one finally gets

$$
\begin{array}{rlrl}
R_{\alpha}(\mathcal{M} ; \boldsymbol{\rho}) & \geq \frac{1}{2} R_{\alpha}(\omega) & (0<\alpha \leq 1) \\
R_{\alpha}(\mathcal{M} ; \boldsymbol{\rho}) \geq \frac{1}{2} R_{\alpha}\left(\omega^{\prime}\right) & (1<\alpha<\infty), \\
R_{\alpha}(\mathcal{M} ; \boldsymbol{\rho}) \geq \frac{1}{1-\alpha} \log \left(\frac{1}{2}+\frac{1}{2} \sum_{i} \omega_{i}^{\alpha}\right) & (1<\alpha<\infty),
\end{array}
$$

The vectors $\omega$ and $\omega^{\prime}$ are calculated due to the described scheme with $c_{1}=f(\eta)^{-1}$,

$$
c_{2}= \begin{cases}\frac{\sqrt{1+2 \eta-3 \eta^{2}}}{1+\eta}, & \text { if } \eta \in[0 ; 0.2], \\ \sqrt{\frac{2-2 \eta}{2-\eta}}, & \text { if } \eta \in[0.2 ; 0.5], \\ \frac{1}{\sqrt{2-\eta}}, & \text { if } \eta \in[0.5 ; 1],\end{cases}
$$

and $c_{3}=1$. Of course, for the Shannon entropy we have $H(\mathcal{M} ; \boldsymbol{\rho}) \geq 0.5 H(\omega)$. For $\alpha>1$, the relations (52) and (53) should be compared. The majorization uncertainty relations are sometimes stronger than the Maassen-Uffink 

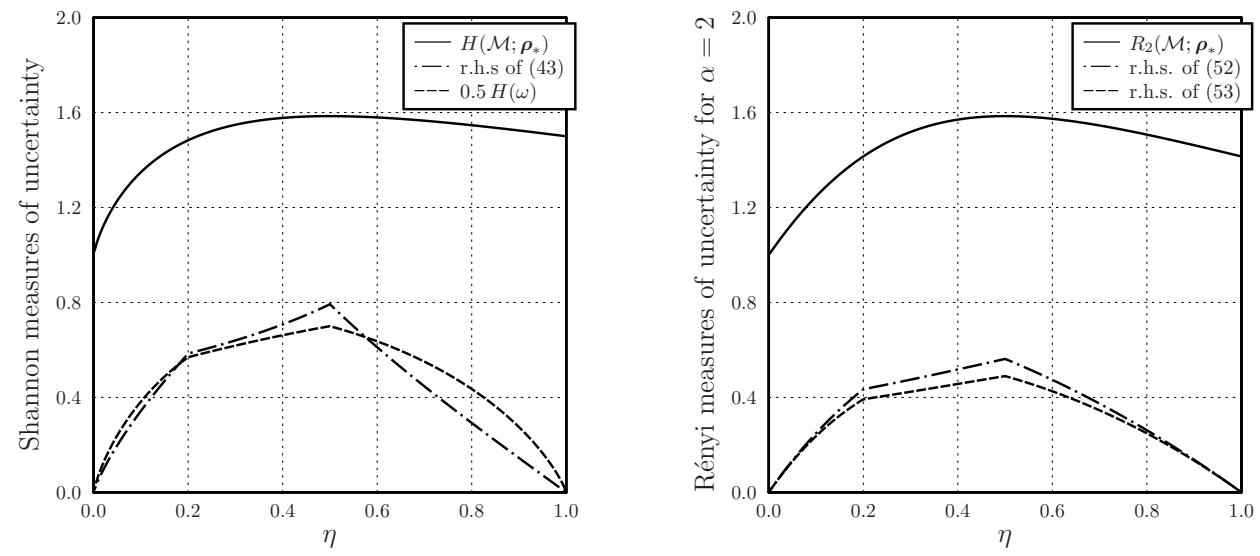

FIG. 1: Comparison of the bounds from entropic uncertainty relations with the corresponding entropies of the completely mixed state $\rho_{*}$ of a qubit.

bound. To illustrate the lower bounds considered, their dependence on $\eta$ is shown in Fig. 1 To estimate the range of possible entropic variations, we also draw the corresponding entropies of the completely mixed state $\boldsymbol{\rho}_{*}=\mathbb{1} / 2$. It is seen on the left plot of Fig. 1 that the relation (43) gives better estimation in a certain range of $\eta$. This fact coincides with some observations reported in [76]. When $\eta$ exceeds 0.6 , the lower bound $0.5 H(\omega)$ from the direct-sum relation is clearly stronger. When $\alpha \neq 1$, we cannot use (40) to estimate from below $R_{\alpha}(\mathcal{M} ; \boldsymbol{\rho})$ solely. The right-hand sides of (52) and (53) both give a desired estimation. For $\alpha=2$, the right-hand side of (52) is slightly better as we see on the right plot of Fig. 1. In general, the curves based on entropic uncertainty relations are not smooth. Apparently, more precise estimation could improve such curves including their asperities. But this will inevitably be related to a kind of direct optimization. The presented lower bounds will further be used for estimating the standard mutual information of Eve from above. It can be expected that the corresponding upper bounds are not smooth in certain points.

\section{MEASURES OF MUTUAL INFORMATION IN THE SCENARIO WITH GENERALIZED DISCRIMINATION}

In this section, we will study the $\alpha$-measures (32)-34) as quantifiers of a performance of the FPB quantum cryptographic probe. The first-type information measure of order $\alpha=2$ was widely used for studying individual attacks [15, 21 23, 43]. For this reason, we begin just with this measure and, throughout, other $\alpha$-measures of the same order $\alpha=2$. By the prime sign, we mean events related to the error-free sifted bits shared by Alice and Bob. In the case under study, one has $I\left(A^{\prime}, B^{\prime}\right)=1-h\left(P_{E}\right)$, where $h\left(P_{E}\right)$ is the binary Shannon entropy. Despite of the fact that $1-h\left(P_{E}\right)=0$ for $P_{E}=0.5$, we will further use the range $P_{E} \in[0 ; 1 / 3]$. These points concern only our particular scenario. Say, security of the BB84 scheme against basis-independent attacks is posed as follows (see, e.g., Theorem 1 of [27]). Secure final key can be extracted from sifted key at the asymptotic rate $\max \{1-2 h(\delta), 0\}$, where $\delta$ is the error rate found in the verification test. The quantity $1-2 h(\delta)$ vanishes, when $\delta$ is about $11.0 \%$ [25]. The latter gives the critical value of the quantum bit error rate for one-way postprocessing without preprocessing [4]. It is sometimes referred to as the Shor-Preskill key rate. If one can use preprocessing or two-way postprocessing, then the corresponding critical values can be increased [4]. However, these values are noticeably less than $1 / 3$. Scenarios with preprocessing or two-way postprocessing are beyond the scope of this work.

Since $\left\langle t_{+} \mid t_{+}\right\rangle=\left\langle t_{-} \mid t_{-}\right\rangle=1-P_{E}$, the angle $\theta \in[0 ; \pi / 4]$ stood in (15) reads as

$$
\cos 2 \theta=\frac{1-3 P_{E}}{1-P_{E}}, \quad \sin 2 \theta=\frac{\sqrt{4 P_{E}\left(1-2 P_{E}\right)}}{1-P_{E}},
$$

The following fact should be emphasized. We will focus on the case, when the legitimate users set the values 0 and 1 to be equally likely. Hence, the binary variable $B^{\prime}$ has the uniform distribution, so that $R_{\alpha}\left(B^{\prime}\right)=\log 2=1$ irrespectively to $\alpha$. For $\alpha>\beta$, we then obtain

$$
I_{\alpha}^{(1)}\left(B^{\prime}, E^{\prime}\right) \geq I_{\beta}^{(1)}\left(B^{\prime}, E^{\prime}\right) .
$$

It follows from the fact that $R_{\alpha}^{(1)}\left(B^{\prime} \mid E^{\prime}\right) \leq R_{\beta}^{(1)}\left(B^{\prime} \mid E^{\prime}\right)$ for $\alpha>\beta$. 
In the scenario with generalized scheme to distinguish $\left|t_{+}\right\rangle$and $\left|t_{-}\right\rangle$, Eve's activity is described by the two parameters $P_{E}$ and $\phi$. For the given $P_{E} \in[0 ; 1 / 3]$, the angle $\phi$ ranges between 0 and $\pi / 4-\theta$. For $j=0,1$, we now write

$$
\begin{aligned}
p\left(e^{\prime}=j \mid b^{\prime}=j\right) & =Q_{S}, \\
p\left(e^{\prime}=0 \mid b^{\prime}=1\right)=p\left(e^{\prime}=1 \mid b^{\prime}=0\right) & =Q_{E}, \\
p\left(e^{\prime}=? \mid b^{\prime}=j\right) & =Q_{?},
\end{aligned}
$$

where the values of $Q_{S}, Q_{E}$, and $Q_{\text {? }}$ are given by (15). Multiplying the above expressions by $1 / 2$, we obtain the corresponding joint probabilities. Hence, we also have

$$
p\left(e^{\prime}=j\right)=\frac{1-Q_{?}}{2}, \quad p\left(e^{\prime}=?\right)=Q ? .
$$

Hence, we obtain $H\left(E^{\prime}\right)=1-Q_{?}+h\left(Q_{\text {? }}\right)$. The final expressions for the conditional probabilities $p\left(b^{\prime} \mid e^{\prime}\right)$ appear as

$$
\begin{aligned}
p\left(b^{\prime}=j \mid e^{\prime}=j\right) & =\frac{Q_{S}}{1-Q_{?}}, \\
p\left(b^{\prime}=0 \mid e^{\prime}=1\right)=p\left(b^{\prime}=1 \mid e^{\prime}=0\right) & =\frac{Q_{E}}{1-Q_{?}}, \\
p\left(b^{\prime}=j \mid e^{\prime}=?\right) & =\frac{1}{2} .
\end{aligned}
$$

Due to (63), one gets $R_{\alpha}^{(1)}\left(B^{\prime} \mid e^{\prime}=?\right)=\log 2=1$. For $R_{\alpha}^{(1)}\left(B^{\prime} \mid e^{\prime}=j\right)$ with $j=0,1$, we obtain more complicated formula. The resulting expression for the first-type $\alpha$-measure of information reads as

$$
I_{\alpha}^{(1)}\left(B^{\prime}, E^{\prime}\right)=\left(1-Q_{?}\right)\left[1-\frac{\log \left(Q_{S}^{\alpha}+Q_{E}^{\alpha}\right)}{1-\alpha}+\frac{\alpha \log \left(1-Q_{?}\right)}{1-\alpha}\right] .
$$

In particular, for $\alpha=\infty$ we have

$$
I_{\infty}^{(1)}\left(B^{\prime}, E^{\prime}\right)=1-Q_{?}-\left(1-Q_{?}\right) \log \left(1-Q_{?}\right)+\left(1-Q_{?}\right) \log Q_{S} .
$$

Further, the standard mutual information is written as

$$
I\left(B^{\prime}, E^{\prime}\right)=1-Q_{?}-\left(1-Q_{?}\right) \log \left(1-Q_{?}\right)+Q_{S} \log Q_{S}+Q_{E} \log Q_{E} .
$$

We prefer to write the above expressions explicitly, since the quantity (64) has widely been used in considering individual attacks.

Using the measures $I_{\alpha}^{(1)}\left(B^{\prime}, E^{\prime}\right), I_{\alpha}^{(2)}\left(B^{\prime}, E^{\prime}\right), I_{\alpha}^{(4)}\left(B^{\prime}, E^{\prime}\right)$ to estimate a performance of the FBP probe, we will compare them with the standard mutual information. Due to [15], the quantity $I_{2}^{(1)}\left(B^{\prime}, E^{\prime}\right)$ was often used for the considered purposes. Since $I_{\infty}^{(1)}\left(B^{\prime}, E^{\prime}\right)$ gives the upper bound due to (56), it will also be shown. With the two schemes of state discrimination, this approach was discussed in [18]. It is useful to compare different information measures in the context of generalized scenarios of discrimination on Eve's side. We will reveal more brightly an inadequacy of $\alpha$-measures of mutual information in the considered context. To do so, we choose several intermediate values of $\phi$ that discretize the allowed interval $0 \leq \phi \leq \pi / 4-\theta$. For definiteness, we will use the characteristic ratio

$$
\xi_{\phi}:=\frac{\phi}{\pi / 4-\theta} .
$$

Its least values 0 and 1 respectively correspond to the unambiguous discrimination and the Helstrom scheme. The parameter (67) characterizes the amount of erroneous outcomes in Eve's results. Further, measures of mutual information will be visualized for $\xi_{\phi}=0.00,0.25,0.50,0.75,1.00$.

Let us begin with the standard mutual information for giving a certain benchmark. In the right plot of Fig. 2, we show $I\left(B^{\prime}, E^{\prime}\right)$ as a function of $P_{E}$ for several values of $\xi_{\phi}$. The value of mutual information is maximal for $\xi_{\phi}=1$, when Eve uses the Helstrom scheme. Eve's mutual information is slightly reduced with decreasing of $\xi_{\phi}$. We see in Fig. 2 that for relatively small values of $P_{E}$ corresponding curves come very close to the curve $\xi_{\phi}=1$. Even in the case of conclusive eavesdropping, Eve's mutual information is quite comparable with this top curve. The latter conclusion was already emphasized in [18]. The maximal difference between the two curves for $\xi_{\phi}=1$ and $\xi_{\phi}=0$ takes place for $P_{E} \approx 0.227$. Noticeable values of the relative difference between curves are observed for negligible 

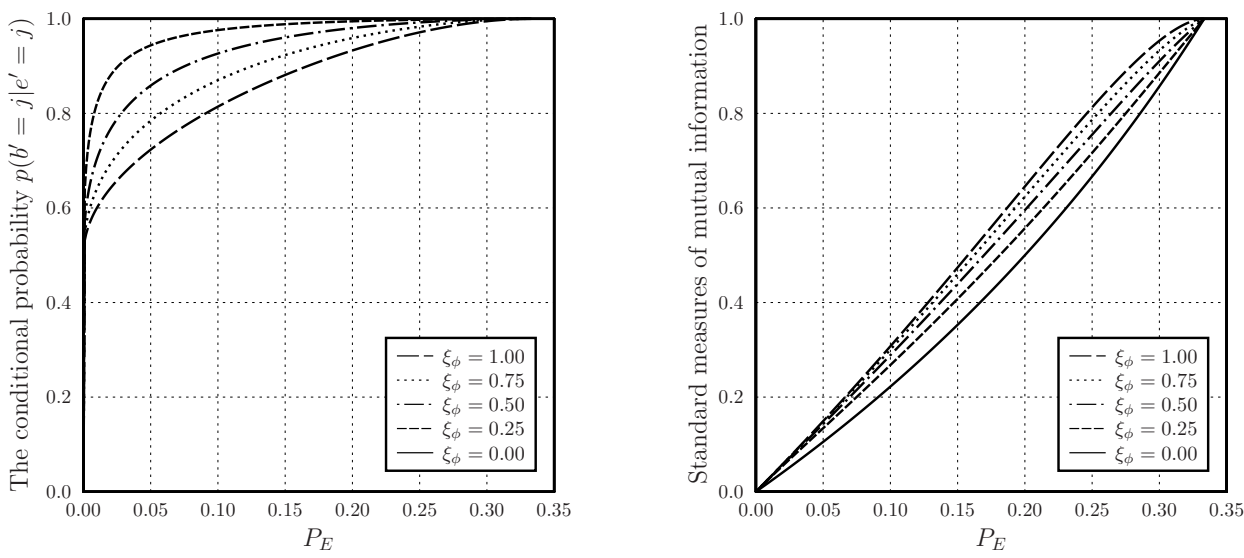

FIG. 2: The conditional probability $p\left(b^{\prime}=j \mid e^{\prime}=j\right)$ and the standard mutual information $I\left(B^{\prime}, E^{\prime}\right)$ versus $P_{E}$ for the five discrimination schemes. As the case $\xi_{\phi}=0$ gives $p\left(b^{\prime}=j \mid e^{\prime}=j\right)=1$, its plot coincides with the top of the left box.
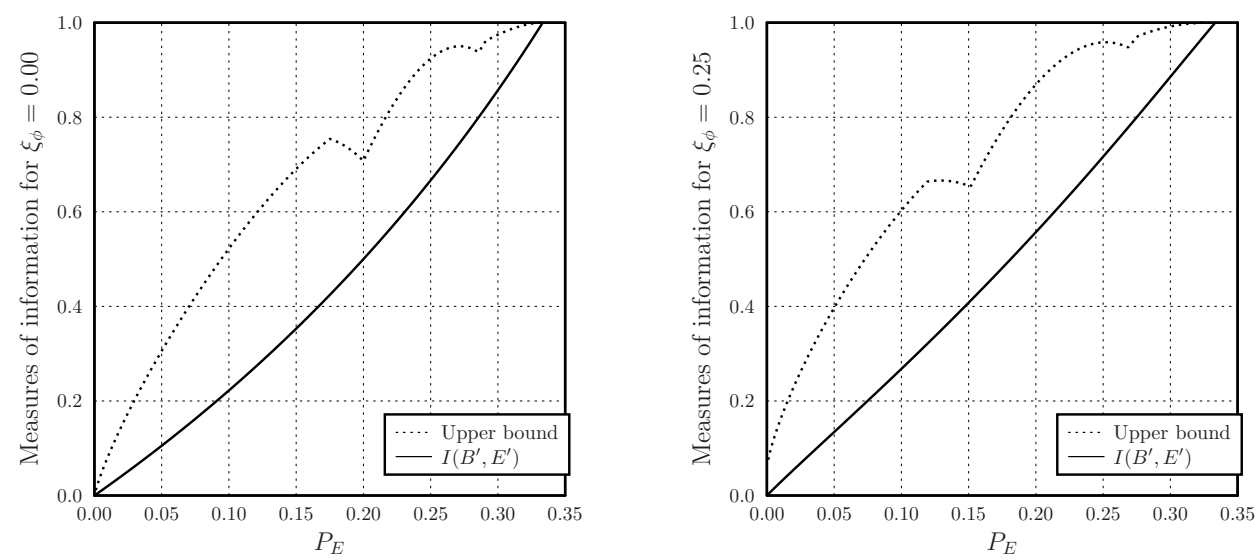

FIG. 3: The standard mutual information $I\left(B^{\prime}, E^{\prime}\right)$ and the right-hand side of (68) versus $P_{E}$.

values of $P_{E}$. Further, we will refer to the considered picture in testing (32)-(34) as characteristics of Eve's mutual information.

Using the uncertainty relation of the previous section, we can estimate from above the standard mutual information $I\left(B^{\prime}, E^{\prime}\right)$. Although this estimate is not not very tight, it will describe a range of possible variations of information measures. Combining (43) with (51) and applying the result to $H\left(E^{\prime} \mid b^{\prime}\right)$ for every $b^{\prime}$, we obtain

$$
I\left(B^{\prime}, E^{\prime}\right) \leq H\left(E^{\prime}\right)-\max \left\{\log f(\eta)+\frac{\eta U(0.2-\eta)}{2(1+\eta)} \log \left(\frac{1-\eta}{4 \eta}\right), \frac{H(\omega)}{2}\right\} .
$$

Here, the parameter $\eta$ characterizes Eve's discrimination scheme. In Fig. 3 we present the mutual information $I\left(B^{\prime}, E^{\prime}\right)$ and the right-hand side of (68) for the two discrimination schemes on Eve's side. The curves are not smooth in certain points. This is originated in non-tightness of the uncertainty relations (43) and (51). Albeit they were optimized with respect to possible Naimark extensions, the tight estimation is still not reached. Probably, an improvement could estimate $I\left(B^{\prime}, E^{\prime}\right)$ more smoothly. Nevertheless, the curves of Fig. 3 demonstrate that a range of possible variations of the mutual information is narrow enough in the studied scenario. It will be instructive to compare Fig. 3 with curves presented in the subsequent figures. At this point, we can recall the Shor-Preskill key rate. Since the term $1-2 h\left(P_{E}\right)$ vanishes for $P_{E} \approx 0.11$, the corresponding estimation from above is not shown as related to other scenarios.

Let us begin with information measures of the form $I_{\alpha}^{(1)}\left(B^{\prime}, E^{\prime}\right)$. As was already mentioned, the measure of information of order $\alpha=2$ has found use in studying a performance of Eve's entangling probes. In the left plot of Fig. 4, we picture $I_{2}^{(1)}\left(B^{\prime}, E^{\prime}\right)$ as a function of $P_{E}$ for the fifth values of $\xi_{\phi}$. First of all, the curve for $\xi_{\phi}=0$ goes just along its track in Fig. 22 Indeed, for conclusive eavesdropping we have $I_{2}^{(1)}\left(B^{\prime}, E^{\prime}\right)=I\left(B^{\prime}, E^{\prime}\right)$. This principal observation made in [18] has lead to the conclusion that the so-called Rényi mutual information is not a completely 

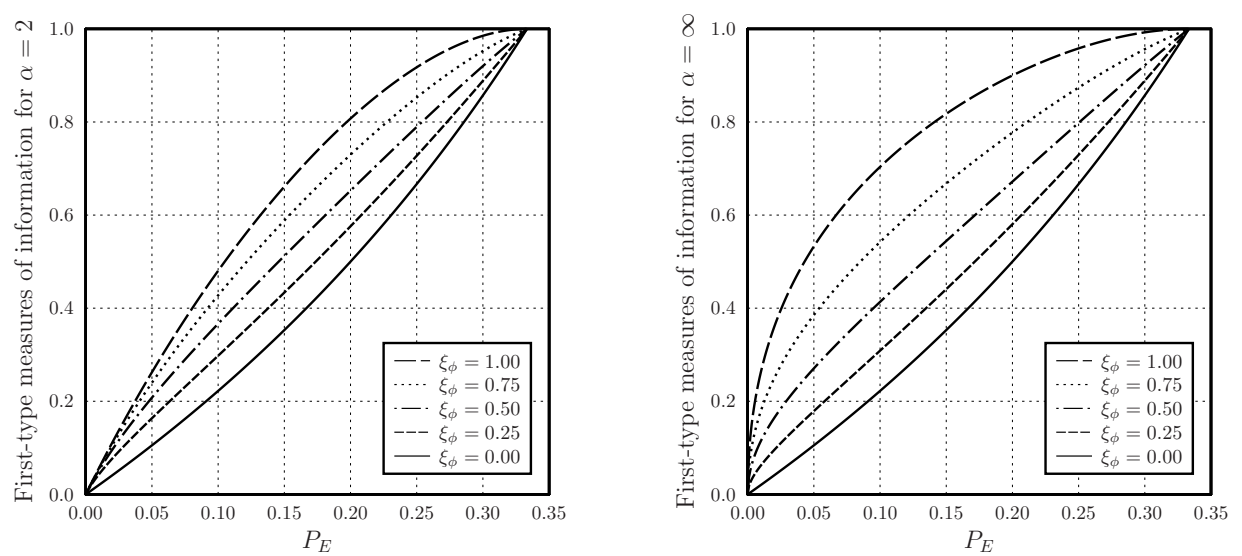

FIG. 4: The first-type measures $I_{2}^{(1)}\left(B^{\prime}, E^{\prime}\right)$ and $I_{\infty}^{(1)}\left(B^{\prime}, E^{\prime}\right)$ versus $P_{E}$ for the five discrimination schemes on Eve's side.

legitimate measure. Using the curves drawn in Fig. 4, we further support the mentioned conclusion. We see in Fig. 2 that the lines of $I\left(B^{\prime}, E^{\prime}\right)$ for intermediate values of $\xi_{\phi}$ are very close to each other. At the same time, the lines of

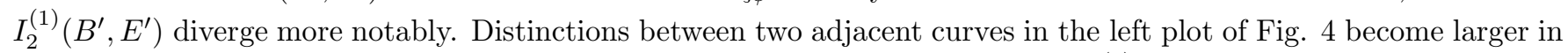
comparison with Fig. 2. The less a fraction of inconclusive answers is, the more $I_{2}^{(1)}\left(B^{\prime}, E^{\prime}\right)$ deviates from $I\left(B^{\prime}, E^{\prime}\right)$. In effect, the mentioned feature concerns even small values of $P_{E}$.

It is instructive to inspect the situation for other values of $\alpha$. In the right plot of Fig. 4, we picture $I_{\infty}^{(1)}\left(B^{\prime}, E^{\prime}\right)$ as a function of $P_{E}$ for the fifth values of $\xi_{\phi}$. Curves for $\alpha=\infty$ are demonstrative due to the property (56). We observe very essential buckling of the curves with a little fraction of inconclusive answers. If our approach is based on the measure (65), then we should accept an essential weakness of scenarios with a large fraction of inconclusive answers. So, we could underestimate the degree of vulnerability with respect to conclusive eavesdropping and close scenarios. As is seen in the plots of Fig. 4, wrong pictures are especially noticeable for relatively small values of $P_{E}$. Dealing with communication security, we cannot be guided by false proposals. At least in the case of individual attacks, $\alpha$-measures of mutual information of the Rényi type are not completely adequate quantifiers. Thus, we have additionally confirmed the fact that the standard mutual information is the only reliable base for estimating quantum-cryptographic probes.

The mentioned distinction is most essential for "weak" eavesdropping, when Eve attempts to get a small amount of information while causing only a slight disturbance. It is sufficiently typical that an opposite party tries to cloak its activity against legitimate users. If we abandon the standard measure, then a quality of the usual FPB probe in comparison with conclusive ones may be illusory up to several times. This example illustrates a conclusion that security restrictions could be overstated spuriously on the base of inadequate measures. Such wrong conclusions about too strong probe performance may lead to rejecting some feasible realizations. They might falsely be evaluated as very vulnerable, even though they are suitable in other respects. As is emphasized in section VI.L of [2], the infinite security will demand the infinite cost. In practice, characteristics of a quantum-cryptographic system are actually determined by some compromise between several conflicting requirements. Hence, we wish to avoid wrong conclusions of any kind.

Let us consider briefly the quantities (33) and (34). They can be treated as candidates to quantify Eve's mutual information about the error-free sifted bits. The case $\alpha=2$ was first be taken concerning an inadequacy of the considered measures to characterize a performance of the FPB probe. With growth of order $\alpha$, similarly inconsistent pictures turned out to be placed. Doubts revealed for $\alpha=2$ will become even more visible. To demonstrate the fact, we shall draw the corresponding curves for $\alpha=10$. This choice is neither better nor worse than others. Rather, it allows us to review a general picture for relatively large values of $\alpha$. In contrast with (32), the quantity (33) is symmetric. If $\alpha$ is very close to 1 , then a picture almost reproduces that is shown in the right plot of Fig. 2 It is not amazing since the right-hand side of (33) aims to simulate the right-hand side of (23). When $\alpha$ deviates from 1 , the corresponding curves are changed essentially. To illustrate this fact, we present the cases $\alpha=2$ and $\alpha=10$ in Fig. 5. The resulting curves reveal a behavior quite different from the curves shown in Figs. 2 and 4 For relatively small values of $\xi_{\phi}=1$, we see an $s$-like shape. That is, for schemes with sufficient number of inconclusive answers the measure (33) is even less suitable than (32). In the left plot of Fig. (5. the lines for $\xi_{\phi}=1$ and $\xi_{\phi}=0.75$ are clearly intersected with an intermediate value $P_{E} \approx 0.108$. No such intersection takes place in Figs. 2 and 4 . More intersections are observed in the right plot of Fig. 5. We finish with the quantity (34) related to more sophisticated version (31) of conditional Rényi's entropy. The cases $\alpha=2$ and $\alpha=10$ are shown in Fig. 6. Although the left plot 

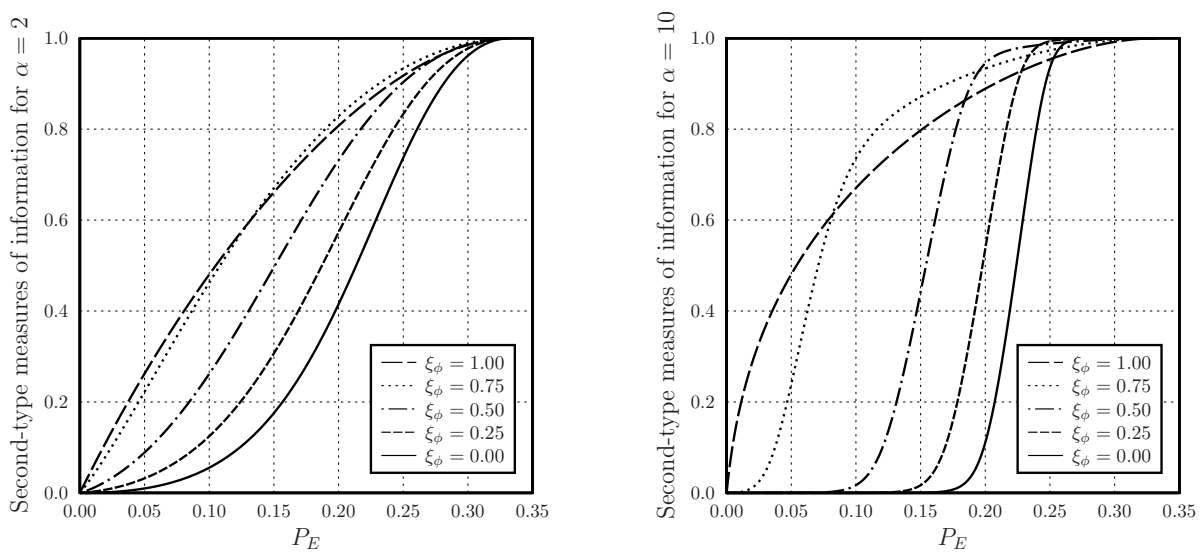

FIG. 5: The second-type measures $I_{2}^{(2)}\left(B^{\prime}, E^{\prime}\right)$ and $I_{10}^{(2)}\left(B^{\prime}, E^{\prime}\right)$ versus $P_{E}$ for the five discrimination schemes on Eve's side.
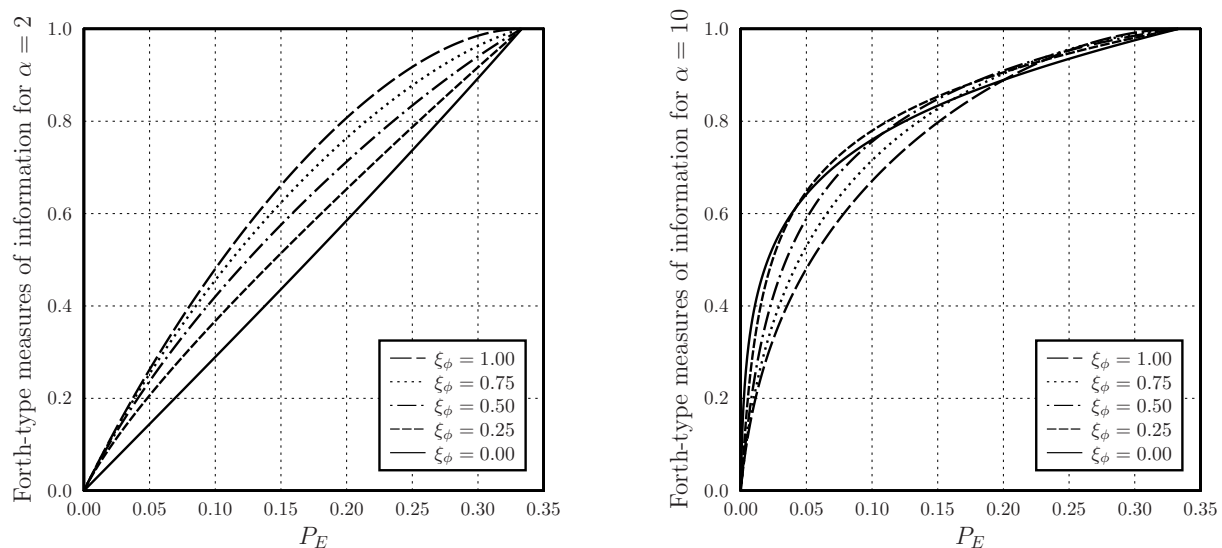

FIG. 6: The forth-type measures $I_{2}^{(4)}\left(B^{\prime}, E^{\prime}\right)$ and $I_{10}^{(4)}\left(B^{\prime}, E^{\prime}\right)$ versus $P_{E}$ for the five discrimination schemes on Eve's side.

of Fig. 6 is similar to the left plot of Fig. 4, the right one is very different. For $\alpha=10$, the lines for various values of $\xi_{\phi}$ intersect many times. These observations witness that the $\alpha$-measures of information are adequate only when $\alpha$ is very close to 1 . However, the latter inevitably implies the use of the standard measure $I\left(B^{\prime}, E^{\prime}\right)$.

Several final remarks concern a possible usage of generalized information functions. The above discussion does not aim to criticize extended information-theoretic functions in general. In effect, generalized entropies were fruitfully used in many important questions. In future, such functions may found novel interesting applications. We only wish to emphasize the necessity of a certain circumspection with their handling in new areas. It is known that generalized entropic functions do not succeed all the properties of the standard functions. For instance, the definition (32) is based on the conditional entropic form, which does not share the chain rule. The authors of 62 have treated the chain rule just as the definition of conditional Rényi's entropy. However, we have above seen an inadequacy of several $\alpha$-measures of mutual information. Other forms of conditional Rényi's entropy, even more sophisticated, will hardly able to improve such things. A certain caution is necessary for dealing with conclusions obtained on the base of generalized information functions.

\section{CONCLUSIONS}

We have examined scenarios with generalized state discrimination after the action of the FPB probe. From this viewpoint, we further studied different quantifiers of an amount of Eve's mutual information about the error-free sifted bits. The so-called Rényi information of order $\alpha=2$ was widely adopted for estimation of a performance of quantum cryptographic probes. It seems that this quantity is not a completely appropriate measure. In the context of individual attacks on the BB84 scheme, wrong conclusions are inspired by means of the first-type 2-measure and related ones. We have also examined another families of information measures inspired by conditional Rényi entropies. It turned out that such measures are even more inadequate from the viewpoint of considered questions. Rather, a 
performance of quantum cryptographic entangling probes is to be evaluated on the base of standard information functions. Using generalized entropies in studies of security of quantum cryptography, we should at least compare various choices of the entropic parameter.

On the other hand, the standard information functions may also be less suitable in some circumstances. In quantum information theory, we have come across numerous questions of different kind. The authors of the papers [77, 78] addressed the problem of properly quantifying informational content of an unknown quantum state. It is natural to adopt mutually unbiased measurements for such purposes. As is shown in [77, 78], the sum of corresponding Shannon entropies has several counter-intuitive properties. More appropriate measure has been proposed and motivated therein. This approach is immediately connected with generalized entropies of order 2 [79]. So, we see that the standard information functions should sometimes be replaced with other functions. A priori, no quantifier can be recognized as the only justified one. Rather, we shall apply several approaches and compare the conclusions obtained. Among existing questions, using the so-called fake states to detect an opposite activity has received less attention than it deserves. In any case, a validity of information measures in application to quantum cryptography should be further studied.

To realize information processing with quantum carriers, real-life systems often deal with imperfect devices. Hence, various security loopholes inevitably occur. The problem of detection-side attacks can be removed by the concept of measurement-device-independent quantum cryptography [80]. Although such attacks are treated as most critical, source-side loopholes should also be removed in practice. For instance, many systems implementing the BB84 protocol realize the source through a weak coherent state with using the decoy-state technique. The authors of [44] described a powerful attack on such systems. It combines the measurement for unambiguous state discrimination with a photonnumber-splitting attack. Here, quantum key distribution without phase randomization can be compromised [44]. Generalized discrimination schemes in source-side attacks on decoy-state quantum cryptography could be the subject of separate investigation.

[1] Lomonaco, S.J.: A quick glance at quantum cryptography. Cryptologia 23, 1-41 (1999)

[2] Gisin, N., Ribordy, G., Tittel, W., Zbinden, H.: Quantum cryptography. Rev. Mod. Phys. 74, 145-195 (2002)

[3] van Assche, G.: Quantum Cryptography and Secret-Key Distillation. Cambridge University Press, Cambridge (2006)

[4] Scarani, V., Bechmann-Pasquinucci, H., Cerf, N.J., Dušek, M., Lütkenhaus, N., Peev, M.: The security of practical quantum key distribution. Rev. Mod. Phys. 81, 1301-1350 (2009)

[5] Nielsen, M.A., Chuang, I.L.: Quantum Computation and Quantum Information. Cambridge University Press, Cambridge (2000)

[6] Galindo, A., Martin-Delgado, M.A.: Information and computation: classical and quantum aspects. Rev. Mod. Phys. 74, $347-423(2002)$

[7] Barnett, S.M.: Quantum Information. Oxford University Press, Oxford (2009)

[8] Shor, P.W.: Polynomial-time algorithms for prime factorization and discrete logarithms on a quantum computer. SIAM J. Comput. 26, 1484-1509 (1997)

[9] Childs, A.M., van Dam, W.: Quantum algorithms for algebraic problems. Rev. Mod. Phys. 82, 1-52 (2010)

[10] Childs, A.M., Ivanyos, G.: Quantum computation of discrete logarithms in semigroups. J. Math. Cryptol. 8, 405-416 (2014)

[11] Bennett, C.H., Brassard, G.: Quantum cryptography: public key distribution and coin tossing. In: Proceedings of IEEE International Conference on Computers, Systems, and Signal Processing, pp. 175-179. IEEE, New York (1984)

[12] Bennett, C.H.: Quantum cryptography using any two nonorthogonal states. Phys. Rev. Lett. 68, 3121-3124 (1992)

[13] Ekert, A.K., Huttner, B., Palma, G.M., Peres, A.: Eavesdropping on quantum-cryptographical systems. Phys. Rev. A 50, 1047-1056 (1994)

[14] Csiszár, I., Körner, J.: Broadcast channels with confidential messages. IEEE Trans. Inf. Theory 24, 339-348 (1978)

[15] Slutsky, B.A., Rao, R., Sun, P.-C., Fainman, Y.: Security of quantum cryptography against individual attacks. Phys. Rev. A 57, 2383-2398 (1998)

[16] Teixeira, A., Matos, A., Antunes, L.: Conditional Rényi entropies. IEEE Trans. Inf. Theory 58, 4273-4277 (2012)

[17] Fehr, S., Berens, S.: On the conditional Rényi entropy. IEEE Trans. Inf. Theory 60, 6801-6810 (2014)

[18] Rastegin, A.E.: On conclusive eavesdropping and measures of mutual information in quantum key distribution. Quantum Inf. Process. 15, 1225-1239 (2016)

[19] Fuchs, C.A., Peres, A.: Quantum state disturbance versus information gain: uncertainty relations for quantum information. Phys. Rev. A 53, 2038-2045 (1996)

[20] Brandt, H.E.: Quantum-cryptographic entangling probe. Phys. Rev. A 71, 042312 (2005)

[21] Brandt, H.E.: Optimum probe parameters for entangling probe in quantum key distribution. Quantum Inf. Process. 2, 37-79 (2003)

[22] Shapiro, J.H.: Performance analysis for Brandt's conclusive entangling probe. Quantum Inf. Process. 5, 11-24 (2006)

[23] Shapiro, J.H., Wong, F.N.C.: Attacking quantum key distribution with single-photon two-qubit quantum logic. Phys. Rev. 
A 73, $012315(2006)$

[24] Herbauts, I.M., Bettelli, S., Hübel, H., Peev, M.: On the optimality of individual entangling-probe attacks against BB84 quantum key distribution. Eur. Phys. J. D 46, 395-406 (2008)

[25] Shor, P.W., Preskill, J.: Simple proof of security of the BB84 quantum key distribution protocol. Phys. Rev. Lett. 85, 441-444 (2000)

[26] Gottesman, D., Lo, H.K.: Proof of security of quantum key distribution with two-way classical communications. IEEE Trans. Inf. Theory 49, 457-475 (2003)

[27] Gottesman, D., Lo, H.K., Lütkenhaus, N., Preskill, J.: Security of quantum key distribution with imperfect devices. Quantum Inf. Comput. 4, 325-360 (2004)

[28] Waks, E., Takesue, H., Yamamoto, Y.: Security of differential-phase-shift quantum key distribution against individual attacks. Phys. Rev. A 73, 012344 (2006)

[29] Vasiliu, E.V.: Non-coherent attack on the ping-pong protocol with completely entangled pairs of qutrits. Quantum Inf. Process. 10, 189-202 (2011)

[30] Bartkiewicz, K., Cernoch, A., Lemr, K., Miranowicz, A., Nori, F.: Temporal steering and security of quantum key distribution with mutually unbiased bases against individual attacks. Phys. Rev. A 93, 062345 (2016)

[31] Biham, E., Boyer, M., Brassard, G., van de Graaf, J., Mor, T.: Security of quantum key distribution against all collective attacks. Algorithmica 34, 372-388 (2002)

[32] Helstrom, C.W.: Detection theory and quantum mechanics. Inf. Control 10, 254-291 (1967)

[33] Helstrom, C.W.: Quantum Detection and Estimation Theory. Academic Press, New York (1976)

[34] Ivanovic, I.D.: How to differentiate between non-orthogonal states. Phys. Lett. A 123, $257-259$ (1987)

[35] Dieks, D.: Overlap and distinguishability of quantum states. Phys. Lett. A 126, 303-306 (1988)

[36] Peres, A.: How to differentiate between non-orthogonal states. Phys. Lett. A 128, 19 (1988)

[37] Huttner, B., Muller, A., Gautier, J.D., Zbinden, H., Gisin, N.: Unambiguous quantum measurement of nonorthogonal states. Phys. Rev. A 54, 3783-3789 (1996)

[38] Chefles, A., Barnett, S.M.: Strategies for discriminating between non-orthogonal quantum states. J. Mod. Opt. 45, 1295$1302(1998)$

[39] Zhang, C.-W., Li, C.-F., Guo, G.-C.: General strategies for discrimination of quantum states. Phys. Lett. A 261, 25-29 (1999)

[40] Fiurášek, J., Ježek, M.: Optimal discrimination of mixed quantum states involving inconclusive results. Phys. Rev. A 67, $012321(2003)$

[41] Matsumoto, R., Watanabe, S.: Key rate available from mismatched mesurements in the BB84 protocol and the uncertainty principle. IEICE Trans. Fundam. 91, 2870-2873 (2008)

[42] Maassen, H., Uffink, J.B.M.: Generalized entropic uncertainty relations. Phys. Rev. Lett. 60, 1103-1106 (1988)

[43] Brandt, H.E.: Unambiguous state discrimination in quantum key distribution. Quantum Inf. Process. 4, 387-398 (2005)

[44] Tang, Y.-L., Yin, H.-L., Ma, X., Fung, C.-H.F., Liu, Y., Yong, H.-L., Chen, T.-Y., Peng, C.-Z., Chen, Z.-B., Pan, J.-W.: Source attack of decoy-state quantum key distribution using phase information. Phys. Rev. A 88, 022308 (2013)

[45] Davies, E.B.: Information and quantum measurement. IEEE Trans. Inf. Theory 24, 596-199 (1978)

[46] Cover, T.M., Thomas, J.A.: Elements of Information Theory. Wiley, New York (1991)

[47] Bennett, C.H., Gács, P., Li, M., Vitányi, P.M.D., Zurek, W.H.: Information distance. IEEE Trans. Inf. Theory 44, 1407-1423 (1998)

[48] Fuchs, C.A., van de Graaf, J.: Cryptographic distinguishability measures for quantum mechanical states. IEEE Trans. Inf. Theory 45, 1216-1227 (1999)

[49] Fuchs, C.A.: Distinguishability and Accessible Information in Quantum Theory. Ph.D. thesis, University of New Mexico, Albuquerque (1996)

[50] Bengtsson, I., Życzkowski, K.: Geometry of Quantum States: An Introduction to Quantum Entanglement. Cambridge University Press, Cambridge (2006)

[51] Devetak, I., Winter, A.: Distillation of secret key and entanglement from quantum states. Proc. R. Soc. A 461, 207-235 (2005)

[52] Acín, A., Brunner, N., Gisin, N., Massar, S., Pironio, S., Scarani, V.: Device-independent security of quantum cryptography against collective attacks. Phys. Rev. Lett. 98, 230501 (2007)

[53] Rényi, A.: On measures of entropy and information. In: Neuman, J. (ed.) Proceedings of 4th Berkeley Symposium on Mathematical Statistics and Probability, vol. 1, pp. 547-561. University of California Press, Berkeley (1961)

[54] Jizba, P., Arimitsu, T.: The world according to Rényi: thermodynamics of multifractal systems. Ann. Phys. 312, 17-59 (2004)

[55] Ben-Bassat, M., Raviv, J.: Rényi's entropy and error probability. IEEE Trans. Inf. Theory 24, 324-331 (1978)

[56] Cachin, C.: Entropy Measures and Unconditional Security in Cryptography. Ph.D. thesis, Swiss Federal Institute of Technology, Zürich (1997)

[57] Kamimura, R.: Minimizing $\alpha$-information for generalization and interpretation. Algorithmica 22, $173-197$ (1998)

[58] Rastegin, A.E.: Convexity inequalities for estimating generalized conditional entropies from below. Kybernetika 48, 242$253(2012)$

[59] Rastegin, A.E.: Further results on generalized conditional entropies. RAIRO-Theor. Inf. Appl. 49, 67-92 (2015)

[60] Zhang, J., Zhang, Y., Yu, C.-S.: Rényi entropy uncertainty relation for successive projective measurements. Quantum Inf. Process. 14, 2239-2253 (2015) 
[61] Rastegin, A.E.: Entropic uncertainty relations for successive measurements of canonically conjugate observables. Ann. Phys. (Berlin) 528, 835-844 (2016)

[62] Golshani, L., Pasha, E., Yari, G.: Some properties of Rényi entropy and Rényi entropy rate. Inf. Sci. 179, 2426-2433 (2009)

[63] Müller-Lennert, M., Dupuis, F., Szehr, O., Fehr, S., Tomamichel, M.: On quantum Rényi entropies: a new generalization and some properties. J. Math. Phys. 54, 122203 (2013)

[64] Wilde, M.M., Winter, A., Yang, D.: Strong converse for the classical capacity of entanglement-breaking and Hadamard channels. Commun. Math. Phys. 331, 593-622 (2014)

[65] Verdu, S.: $\alpha$-mutual information. In: Workshop on Information Theory and Applications, San-Diego, California, USA, February. http://ita.ucsd.edu/workshop/15/files/paper/paper374.pdf (2015)

[66] Shao, L.-H., Li, Y., Luo, Y., Xi, Z.: Quantum coherence quantifiers based on the Rényi $\alpha$-relative entropy. Commun. Theor. Phys. 67, 631-636 (2017)

[67] Xu, J.: Coherence measures based on sandwiched Rényi relative entropy. E-print arXiv:1808.04662 [quant-ph] (2018)

[68] Deutsch, D.: Uncertainty in quantum measurements. Phys. Rev. Lett. 50, 631-633 (1983)

[69] Wehner, S., Winter, A.: Entropic uncertainty relations - a survey. New J. Phys. 12, 025009 (2010)

[70] Coles, P.J.; Berta, M., Tomamichel, M., Wehner, S.: Entropic uncertainty relations and their applications. Rev. Mod. Phys. 89, 015002 (2017)

[71] Kraus, K.: Complementary observables and uncertainty relations. Phys. Rev. D 35, 3070-3075 (1987)

[72] Massar, S.: Uncertainty relations for positive-operator-valued measures. Phys. Rev. A 76, 042114 (2007)

[73] Coles, P.J., Piani, M.: Improved entropic uncertainty relations and information exclusion relations. Phys. Rev. A 89, 022112 (2014)

[74] Z. Puchała, Ł. Rudnicki, and K Życzkowski, Majorization entropic uncertainty relations. J. Phys. A: Math. Theor. 46, 272002 (2013)

[75] S. Friedland, V. Gheorghiu, and G. Gour, Universal uncertainty relations, Phys. Rev. Lett. 111, 230401 (2013)

[76] Ł. Rudnicki, Z. Puchała, and K. Życzkowski, Strong majorization entropic uncertainty relations. Phys. Rev. A 89, 052115 (2014)

[77] Brukner, С̆., Zeilinger, A.: Operationally invariant information in quantum measurements. Phys. Rev. Lett. 83, 3354-3357 (1999)

[78] Brukner, C̆., Zeilinger, A.: Conceptual inadequacy of the Shannon information in quantum measurements. Phys. Rev. A 63, 022113 (2001)

[79] Rastegin, A.E.: On the Brukner-Zeilinger approach to information in quantum measurements. Proc. R. Soc. A 471, 20150435 (2015)

[80] Lo, H.-K., Curty, M., Qi, B.: Measurement-device-independent quantum key distribution. Phys. Rev. Lett. 108, 130503 (2012) 